\title{
ERRATUM
}

\section{Almonds vs complex carbohydrates in a weight reduction program}

MA Wien, JM Sabaté, DN Iklé and FR Kandeel

International Journal of Obesity (2004) 28, 459. doi:10.1038/sj.ijo.0802607

Correction to: International Journal of Obesity (2003) 27, 1365-1372. doi: 10.1038/sj.ijo.0802411

In the abstract of the above paper, the last sentence in the results section was incorrectly published. The sentence should read:
Among subjects with type 2 diabetes, diabetes medication reductions were sustained or further reduced in a greater proportion of almond-LCD as compared to CHO-LCD subjects (96 vs 50\%, respectively). 